\title{
ИНТЕРАКТИВНЫЙ МЕТОД РАСЧЕТА ТЕПЛООБМЕННЫХ АППАРАТОВ С УЧЕТОМ ФАЗОВЫХ ПЕРЕХОДОВ
}

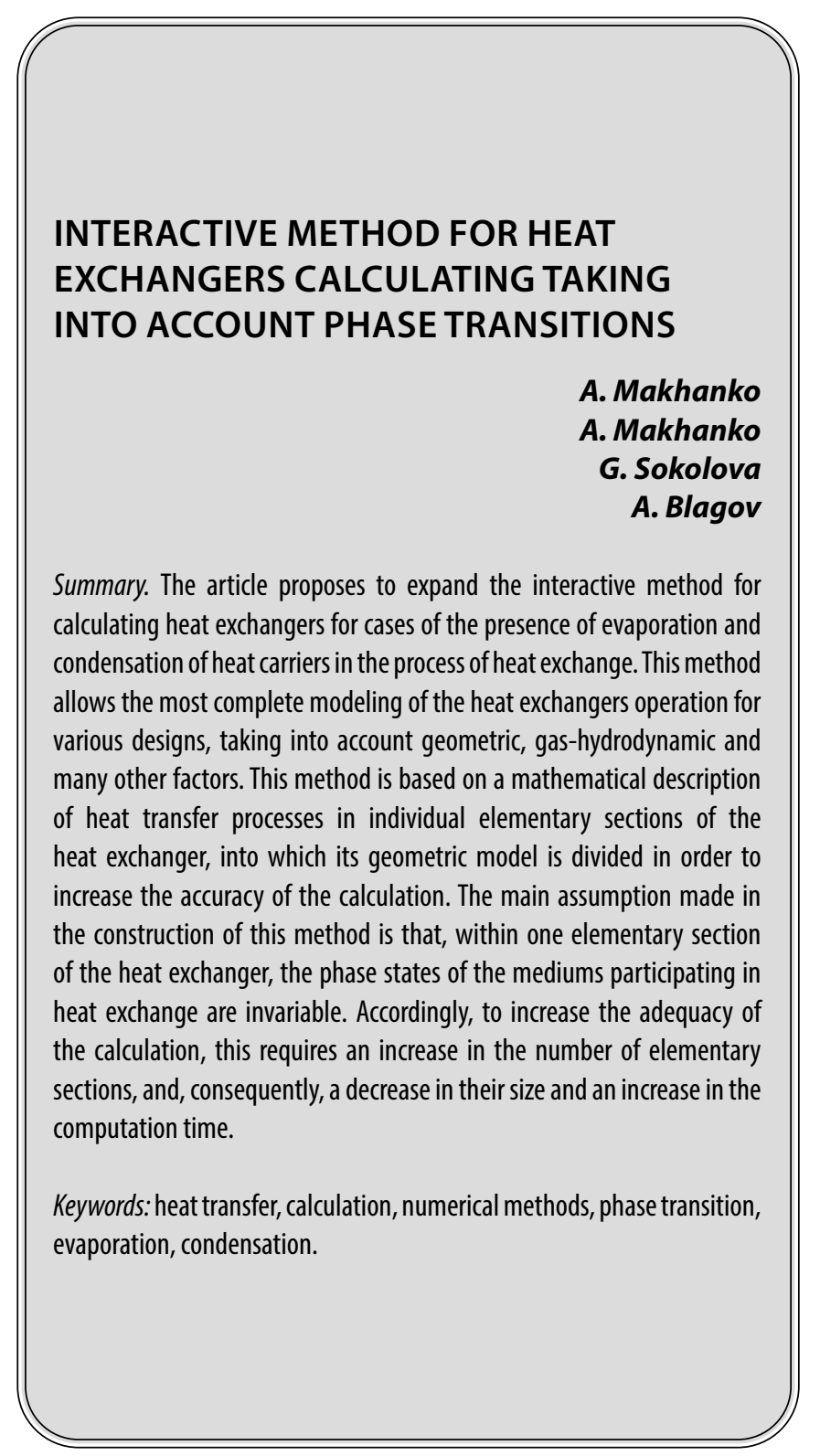

\section{Введение}

B современной теплотехнике большое распространение получили теплообменные аппараты разных видов [8, с. 2831] [2, с. 54]. Особую сложность в расчёте таких аппаратов представляют ситуации, когда теплообмен в них сопровождается не только падением или нарастанием температуры однофазной среды, а кроме этого, происходят ещё и фазовые переходы (испарение, конденсация) [3, с. 207] [4, с. 58].
Маханько Анатолий Васильевич

К.т.н., дочент, Казанский национальный исследовательский технический университет - КАИ им. А. Н. Туполева

Маханько Андрей Анатольевич

К.т.н., дочент, Казанский начиональный исследовательский технический университет - КАИ им. А. Н. Туполева andmahanko@mail.ru Соколова Галина Павловна

К.n.н., дочент, Казанский национальный исследовательский технический университет - КАИ им. А. Н. Туполева glnsokolova@mail.ru

Благов Алексей Евгеньевич

Старший преподаватель, Казанский начиональный исследовательский технический университет - КАИ им. А. Н. Туполева bae311265@mail.ru

Аннотация. В статье предлагается расширить интерактивный метод расчета теплообменников для случаев присутствия в процессе теплообмена испарения и конденсации теплоносителей. Такой метод позволяет наиболее полно моделировать работу теплообменных аппаратов различной конструкции с учетом геометрических, газо- гидродинамических и многих других факторов. Основу метода составляет математическое описание процессов теплообмена на отдельных элементарных участках теплообменного аппарата, на которые разделяется его геометрическая модель в целях повышения точности расчета. Основным допущением, сделанным при построении данного метода, является то, что в пределах одного элементарного участка теплообменника фазовые состояния сред, участвующих в теплообмене, неизменно. Соответственно, для повышения адекватности расчета это требует увеличения количества элементарных участков, а, следовательно, уменьшения их размера и увеличения времени счета.

Ключевые слова: теплообмен, расчет, численные методы, фазовый переход, испарение, конденсация.

В работе «Интерактивный метод расчета теплообменных аппаратов» [5, с. 34] уже рассмотрен итеративный метод расчета теплообменников без фазовых переходов. Этот метод позволяет рассчитывать теплообменники, у которых по длине могут изменяться свойства - сечение труб, толщина стенок труб, давление в трубах и прочие. Метод, предложенный в указанной статье, в случае расчета теплообменника с противотоком требует итеративную процедуру, поскольку начальные температуры при расчете задаются на противоположных сечениях 
теплообменника. Так как в схеме расчета анализируются процессы на отдельных малых участках теплообменника, то метод может быть расширен на случай, когда на отдельных участках каналов теплообменника может происходить конденсация или испарение среды.

\section{Возможные режимы и анные}

Алгоритм расчета при отсутствии фазовых переходов в теплообменнике рассмотрен в работе «Интерактивный метод расчета теплообменных аппаратов» [5, с. 34]. Для расчета теплообменника применим схему, позволяющую рассматривать теплообменники с фазовым переходом в процессе теплообмена и различными свойствами по длине теплообменника.

В процессе расчёта аналогично ранее предложенному методу [5, с. 35] теплообменник разбивается на отдельные участки, в пределах которых сочетания фазовых процессов можно считать неизменными. К возможным сочетаниям фазовых процессов можно отнести такие как: охлаждение обогревающего контура (в жидкой или газообразной фазе)) - испарение в обогреваемом контуре, конденсация в обогревающем контуре - однофазный нагрев в обогреваемом контуре (жидкость или газ), конденсация в обогревающем контуре - испарение в обогреваемом контуре. Для корректного расчёта на каждом таком участке необходимо ввести следующие параметры:

$\mathrm{LL}$-длина теплообменника (м),

dcp - средний диаметр внутренней трубы теплообменника (м),

Fтo=3,14*dcp*LL -площадь поверхности теплообме$\mathrm{Ha}\left(\mathrm{M}^{2}\right)$,

$\delta$ - толщина стенки трубы (м),

$\lambda$ - теплопроводность материала трубы,

Q10, Q1k- тепловая мощность на входе и выходе нагревающего теплоносителя (дж/с),

Q20, Q2k- тепловая мощность на входе и выходе нагреваемого теплоносителя (дж/с),

T10, T1k - температура на входе и выходе нагревающего теплоносителя (K),

T20, T2k - температура на входе и выходе нагреваемого теплоносителя (K),

$\mathrm{dm} 1 / \mathrm{dt}$ - массовый расход нагревающего теплоносителя (кг/c)

$\mathrm{dm} 2 / \mathrm{dt}$ - массовый расход нагреваемого теплоносителя (кг/с)

c1, с2 - удельные теплоемкости нагревающего и нагреваемого теплоносителя (дж/(кг*K)),

a1, a2 - коэффициенты теплоотдачи между стенкой трубы и теплоносителем (вт/(M*K)).

При расчетах процессов теплообмена с фазовыми переходами необходимо добавить зависимость теплоты конденсации от температуры $L_{\kappa о H}(T)$ при заданном давлении [10, с. 398], как для нагревающей среды, так и для нагреваемой.

По известным геометрическим (LL, dcp, Fto $=3,14^{*} \mathrm{~d}$ cp*LL) и теплофизическим свойствам теплоносителей (c1, с2) надо найти зависимость выходной температуры нагреваемого канала Т2k от входной температуры нагревающего канала Т10 и массовых расходов dm1/dt и dm2/dt.

Чтобы получить инструмент [9, с. 98], позволяющий рассчитывать теплообмен как без фазовых переходов, так и с фазовыми переходами, применим итеративную схему вычислений.

\section{$\mathrm{KoH} \Delta$ енсашия в обогреваюшем контуре - о ннофазный нагрев в обогреваемом контуре}

Рассмотрим случай, когда на выбранном участке $\delta x$ температура теплоносителя в нагревательном канале в ходе его охлаждения достигла температуры конденсации при давлении, присутствующем в этом канале. В процессе конденсации температура среды остается постоянной и равной температуре конденсации $\mathrm{T}_{\text {КОН }}$ (рис. 1). При конденсации выделяется тепловая энергия (тепловая мощность)

$$
\begin{aligned}
& \Delta Q_{\text {КОН }}=\frac{d m 1_{K O H}}{d t} * L 1_{\text {КОН }} \quad \text { (дж/с=вт) } \\
& \text { где } \frac{d m 1_{K O H}}{d t}-
\end{aligned}
$$

массовая скорость конденсации (кг/с), $L 1_{\text {KоH }}-$ теплота конденсации (дж/кг).

Поскольку теперь в нагревательном канале присутствуют одновременно две фазы - газообразная и жидкая, введем две величины

$$
\frac{d m 1_{\Gamma}}{d t} и \frac{d m 1_{\mathcal{K}}}{d t}
$$

соответствующие массовым расходам газа и жидкости на входе участка $\delta$. Нахождение связи между массовым расходом и скоростью течения представляет серьёзную задачу требующую специальной подготовки в газо- и гидродинамике [6, с. 97] [9, с. 96] связанную с учетом режима движения [1, с. 197] [7, с. 11].

С учетом конденсации на выходе участка получим расходы 


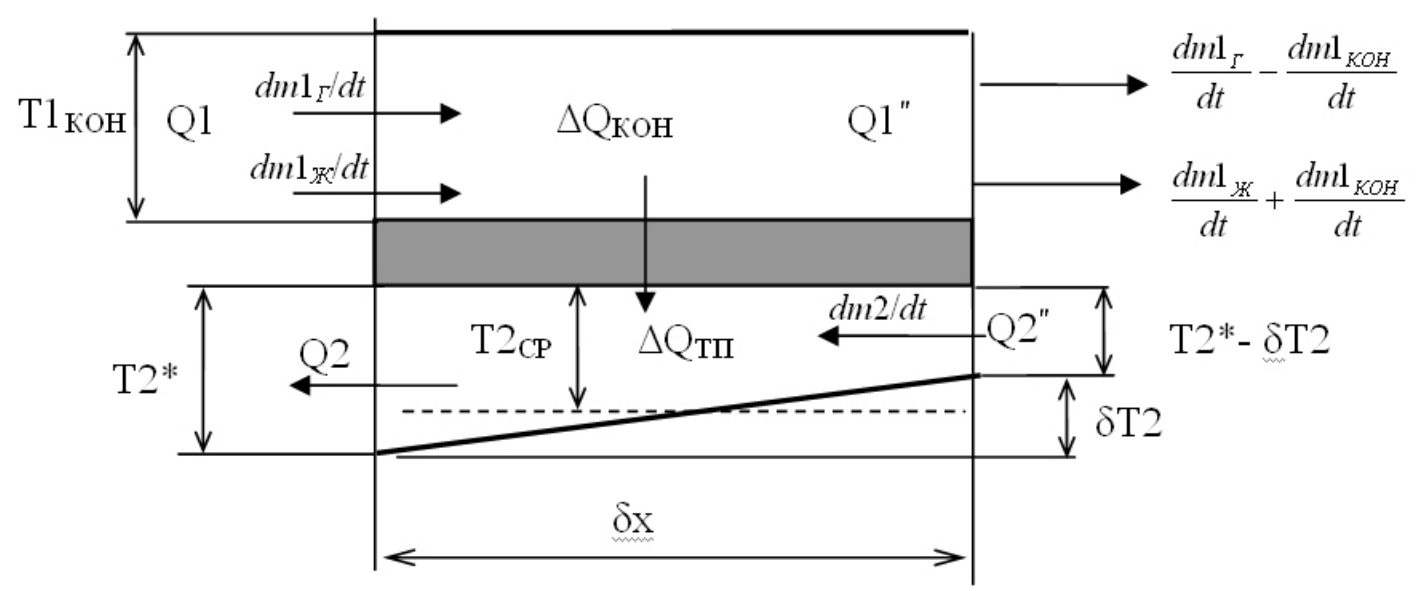

Рис. 1. Схема тепловых процессов

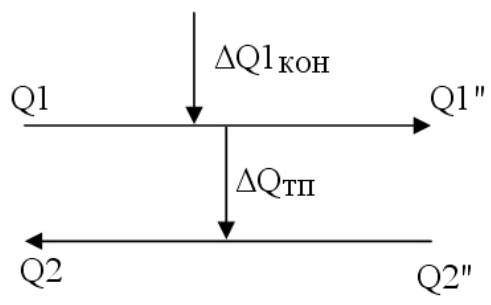

Рис. 2. Схема энергетических процессов

$$
\frac{d m 1_{\Gamma}}{d t}-\frac{d m 1_{K O H}}{d t} \frac{d m 1_{\mathcal{X}}}{d t}+\frac{d m 1_{K O H}}{d t}
$$

Схема тепловых процессов в элементе $\delta x$ показана на рисунке 1.

Средняя температура в нагреваемом канале

$$
T 2_{C P}=T 2 *-\frac{\delta T 2}{2} \text {. }
$$

Баланс энергий нагревающего канала (рис. 2) $Q 1^{\prime \prime}=Q 1+\Delta Q 1_{K O H}-\Delta Q_{T \Pi}$

Баланс энергий нагреваемого канала (рис. 2)

$Q 2=Q 2^{\prime \prime}+\Delta Q_{T \Pi}$

Значения соответствующих выражений примут вид

$$
\begin{aligned}
& Q 1=\left(c 1_{\Gamma} \frac{d m 1_{\Gamma}}{d t}+c 1_{Ж} \frac{d m 1_{Ж}}{d t}\right) T 1_{K O H}, \\
& Q 1 "=\left(c 1_{\Gamma}\left(\frac{d m 1_{\Gamma}}{d t}-\frac{d m 1_{K O H}}{d t}\right)+\right. \\
& \left.+c 1_{Ж}\left(\frac{d m 1_{Ж}}{d t}+\frac{d m 1_{K O H}}{d t}\right)\right) T 1_{K O H}
\end{aligned}
$$

$$
\begin{aligned}
& \Delta Q 1=Q 1-Q 1^{\prime \prime}=\left(c 1_{\Gamma}-c 1_{Ж}\right) \frac{d m 1_{K O H}}{d t} T 1_{K O H}(1) \\
& \Delta Q_{T \Pi}=s k\left(T 1_{K O H}-T 2_{C P}\right)= \\
& =s k\left(T 1_{K O H}-T 2 *+\frac{\delta T 2}{2}\right) \\
& Q 2=c 2 \frac{d m 2}{d t} T 2 *, Q 2^{\prime \prime}=c 2 \frac{d m 2}{d t}(T 2 *-\delta \mathrm{T} 2), \\
& \Delta Q 2=Q 2-Q 2^{\prime \prime}=c 2 \frac{d m 2}{d t} \delta \mathrm{T} 2
\end{aligned}
$$

Поскольку для большинства веществ величина $\mathrm{c1}$ ж $>\mathrm{c1}_{\Gamma}$, получаем (1) $\Delta Q 1<0$, т.е. Q1»>Q1 - на выходе нагревательного канала тепловой энергии больше, чем на входе за счет большей суммарной теплоемкости при постоянстве температуры и не вся энергия конденсации передается через стенку в нагреваемый канал. Если для некоторого вещества $\mathrm{c} 1_{ж}=\mathrm{c} 1_{\Gamma}$, теплосодержание на выходе канала равно теплосодержанию на входе и, следовательно, вся энергия конденсации передается в нагреваемый канал через теплопроводность стенки.

В задаче присутствуют две неизвестные величины 


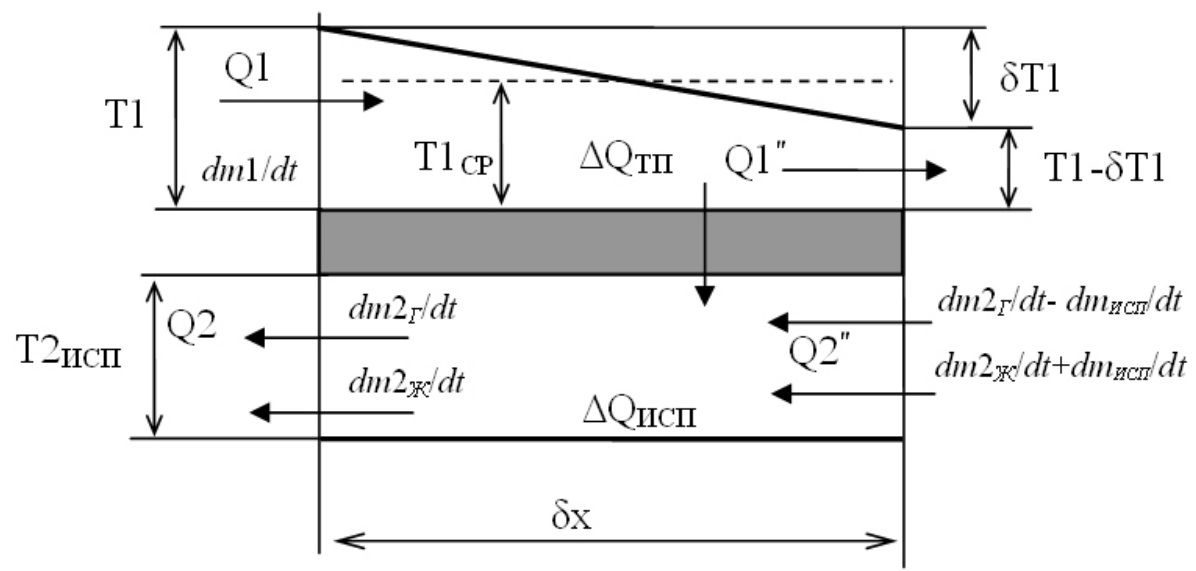

Рис. 3. Схема тепловых процессов

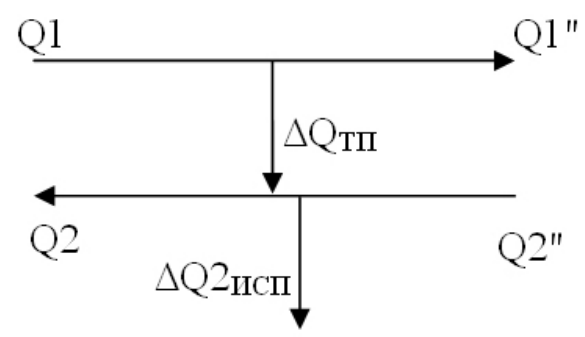

Рис. 4. Схема энергетических процессов

$\delta T 2$ и $\frac{d m 1_{K O H}}{d t}$

Из соотношений (1) получим

$\Delta Q 1=\Delta Q 1_{K O H}-\Delta Q_{T \Pi}$

$\Delta Q 2=\Delta Q_{T \Pi}$

После соответствующих подстановок

$$
\begin{aligned}
& \left(c 1_{\Gamma}-c 1_{\text {Ж }}\right) \frac{d m 1_{K O H}}{d t} T 1_{K O H}=\frac{d m 1_{K O H}}{d t} L 1_{K O H}- \\
& -\operatorname{sk}\left(\mathrm{T} 1_{\mathrm{KOH}}-\mathrm{T} 2 *+\delta \mathrm{T} 2 / 2\right)
\end{aligned}
$$

$c 2 \frac{d m 2}{d t} \delta \mathrm{T} 2=\operatorname{sk}\left(\mathrm{T}_{1 \mathrm{KOH}}-\mathrm{T} 2 *+\delta \mathrm{T} 2 / 2\right)$

Выражение (2) представим в виле системы двух уравнений относительно неизвестных $\delta T 2$ и

$$
\begin{aligned}
& \frac{d m 1_{K O H}}{d t} . \\
& \left(\left(c 1_{\Gamma}-c 1_{\text {Ж }}\right) T 1_{K O H}-L 1_{K O H}\right) \frac{d m 1_{K O H}}{d t}+
\end{aligned}
$$

$$
\begin{aligned}
& +\mathrm{sk} \cdot \delta \mathrm{T} 2 / 2=-\mathrm{sk}\left(\mathrm{T} 1_{\mathrm{KOH}}-\mathrm{T} 2 *\right) \\
& \left(c 2 \frac{d m 2}{d t}-\frac{\mathrm{sk}}{2}\right) \cdot \delta \mathrm{T} 2=\operatorname{sk}\left(\mathrm{T} 1_{\mathrm{\kappa OH}}-\mathrm{T} 2 *\right)
\end{aligned}
$$

\section{Отсюда}

$$
\begin{aligned}
& \delta \mathrm{T} 2=\frac{\mathrm{sk}\left(\mathrm{Tk}_{\mathrm{KOH}}-\mathrm{T} 2 *\right)}{\left(c 2 \frac{d m 2}{d t}-\mathrm{sk} / 2\right)} \\
& \frac{d m 1_{K O H}}{d t}=\frac{s k\left(T 2 *-\delta T 2 / 2-T 1_{K O H}\right)}{\left(\left(c 1_{\Gamma}-c 1_{Ж}\right) T 1_{K O H}-L 1_{K O H}\right)}
\end{aligned}
$$

В нижней формуле (4) -sk учтено в знаках $T 2 *$ и $T 1_{K O H}$

В результате расчета на участке $\delta$ п получаем значения

$$
\frac{d m 1_{\Gamma}}{d t}-\frac{d m 1_{K O H}}{d t}, \frac{d m 1_{Ж}}{d t}+\frac{d m 1_{K O H}}{d t} \text { и } T 2 *-\delta T 2,
$$

которые принимаются за исходные значения на следующем участке. Если на рассматриваемом участке весь газ сконденсировался, для следующих участков применяются другие формулы расчета. 
Охлажление обогреваюшего

контура - испарение

в обогреваемом контуре

Если в нагреваемом канале на участке $\delta$ температура достигнет температуры испарения $\mathrm{T}_{\text {Исп }}$ (рис. 3) при том давлении, которое существует в канале, температура в нагреваемом канале на этом участке будет оставаться постоянной.

При испарении поглощается тепловая энергия (тепловая мощность) (рис. 4)

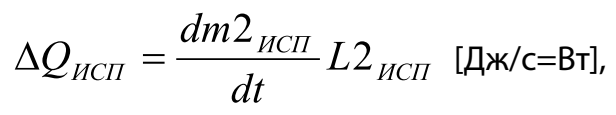

где $\frac{d m 2_{\text {исп }}}{d t}-$

скорость испарения на участке $\delta x$.

Остальные соотношения аналогичны предыдущим вариантам:

$$
\begin{aligned}
& \frac{d m 2_{\text {ИСП }}}{d t}, Q 1 "=c 1 \frac{d m 1}{d t}(T 1-\delta \mathrm{T} 1), \\
& \Delta Q 1=Q 1-Q 1^{\prime \prime}=c 1 \frac{d m 1}{d t} \delta \mathrm{T} 1
\end{aligned}
$$

Поскольку по принципу организации итеративного вычисления известными принимаются параметры процесса на левой границе участка $\delta x$, соотношения массовых расходов газа и жидкости определяются в виде:

на левой границе $d m 2_{\Gamma} / d t \mathbf{n} d m 2_{ж} / d t$

на правой границе $d m 2_{\Gamma} / d t-d m_{\text {ИСI }} / d t$

и $d m 2_{Ж} / d t+d m_{\text {ИСП }} / d t$.

При том получим

$Q 2=\left(c 2_{\Gamma} \frac{d m 2_{\Gamma}}{d t}+c 2_{Ж} \frac{d m 2_{Ж}}{d t}\right) T 2_{\text {исп }}$

$Q 2 "=\left(c 2_{\Gamma}\left(\frac{d m 2_{\Gamma}}{d t}-\frac{d m 2_{\text {ИСП }}}{d t}\right)+\right.$

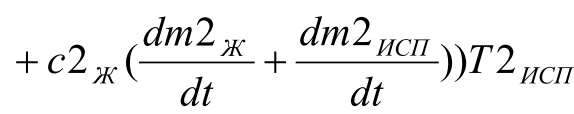

$\Delta Q 2=Q 2-Q 2^{\prime \prime}=\left(-c 2_{Г}+c 2_{\text {ж }}\right) \frac{d m 2_{\text {исп }}}{d t} T 2_{\text {исп }}$
Отметим, что при $\mathrm{c2} 2_{ж}>\mathrm{c2}{ }_{\Gamma}$, получаем $\Delta Q 2<0$, т.е. на выходе нагреваемого канала тепловой энергии меньше, чем на входе за счет испарения

$$
\Delta Q_{\text {ТП }}=\operatorname{sk}\left(T 1_{С Р}-T 2_{\text {ИСП }}\right)=, \operatorname{sk}\left(T 1-\delta T / 2-T 2_{\text {иСП }}\right)
$$

В задаче присутствуют две неизвестные величины

$\delta T 1$ и $\frac{d m 2_{\text {ИСП }}}{d t}$

Из соотношений (5) получим

$\Delta Q 1=\Delta Q_{T \Pi}$

$\Delta Q 2=\Delta Q_{T \Pi}-\Delta Q 2_{\text {иСП }}$

После соответствующих подстановок

$$
\begin{aligned}
& c 1 \frac{d m 1}{d t} \delta \mathrm{T} 1=\operatorname{sk}\left(\mathrm{T} 1-\delta \mathrm{T} 1 / 2-\mathrm{T} 2_{\text {исп }}\right) \\
& \left(-c 2_{Г}+c 2_{ж}\right) \frac{d m 2_{\text {исп }}}{d t} T 2_{\text {исп }}=
\end{aligned}
$$

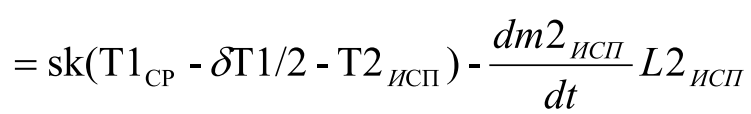

Из выражения (6) можно получить систему двух уравнений относительно неизвестных

$$
\begin{aligned}
& \delta T 1 \text { и } \frac{d m 2_{\text {исп }}}{d t} . \\
& \left(c 1 \frac{d m 1}{d t}+\mathrm{sk} / 2\right) \cdot \delta \mathrm{T} 1=\operatorname{sk}\left(\mathrm{T} 1-\mathrm{T} 2_{\text {исп }}\right) \\
& \left(\left(-c 2_{\Gamma}+c 2_{\text {ж }}\right) T 2_{\text {исп }}+L 2_{\text {иСп }}\right) \frac{d m 2_{\text {ИСП }}+}{d t}+ \\
& +\mathrm{sk} \cdot \delta \mathrm{T} 1 / 2=\operatorname{sk}\left(\mathrm{T} 1-\mathrm{T} 2_{\text {исп }}\right)
\end{aligned}
$$

\section{Отсюда}

$$
\begin{aligned}
& \delta T 1=\frac{\mathrm{sk}\left(\mathrm{T} 1-\mathrm{T} 2_{\text {исп }}\right)}{c 1 \frac{d m 1}{d t}+\mathrm{sk} / 2} \\
& \frac{d m 2_{\text {иСП }}}{d t}=\frac{\operatorname{sk}\left(\mathrm{T} 1-\delta \mathrm{T} 1 / 2-\mathrm{T} 2_{\text {исп }}\right)}{\left(-c 2_{Г}+c 2_{ж}\right) T 2_{\text {исп }}+L 2_{\text {исп }}}
\end{aligned}
$$




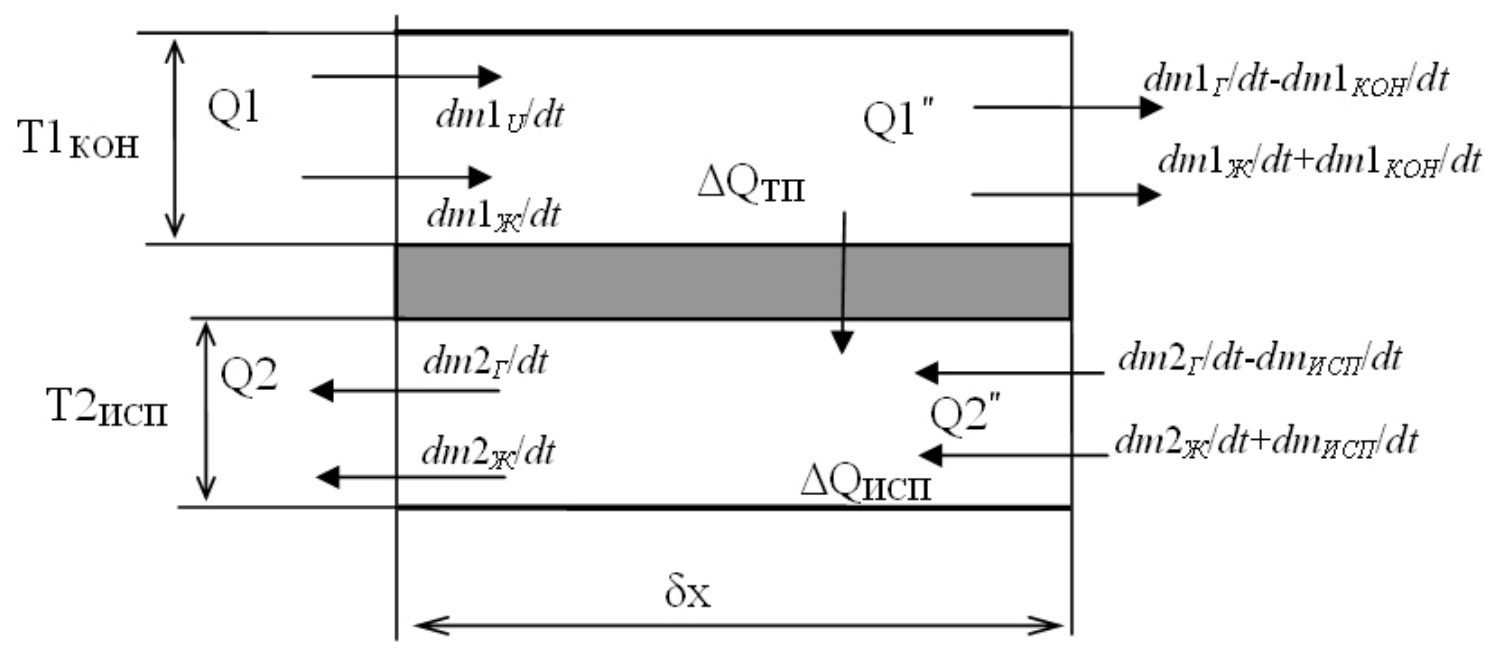

Рис. 5. Схема тепловых процессов

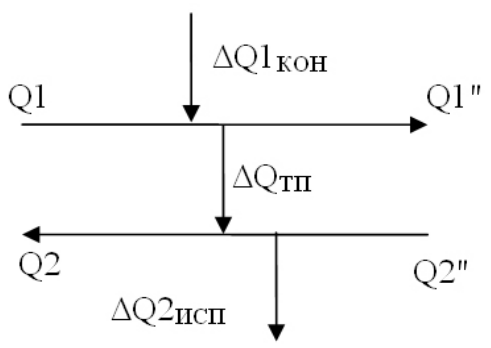

Рис. 6. Схема энергетических процессов

Аналогично предыдущему случаю мы получаем приращения параметров на рассчитываемом участке теплообменника, которые позволяют определить начальные условия для расчета, следующего участка.

\section{Конденсашия в обогреваюшем контуре - испарение \\ в обогреваемом контуре}

В этом случае на участке $\delta x$ температура в обоих каналах будет оставаться постоянной $T_{\text {КОН }}$ и $T 2_{\text {исп }}$ (рис. 5) определимым из условий работы теплообменника, в первую очередь давлением в обогреваемом и обогревающем контурах. Следовательно, перед началом расчета необходимо каким-то образом определить (из внешних условий) рабочие давления обоих контуров.

При испарении поглощается тепловая энергия (тепловая мощность) (рис. 6)

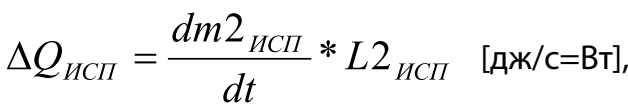

$$
\begin{aligned}
& \text { где } \frac{d m 2_{\text {ИСП }}}{d t}-
\end{aligned}
$$

скорость испарения на участке $\delta x$.

При конденсации выделяется тепловая энергия (тепловая мощность)

$$
\Delta Q_{K O H}=\frac{d m 1_{K O H}}{d t} * L 1_{K O H} \quad[\mathrm{~A} / \mathrm{c}=\mathrm{BT}]
$$

Остальные соотношения аналогичны предыдущим вариантам:

В задаче присутствуют две неизвестные величины

$$
\begin{aligned}
& \frac{d m 1_{\text {КОН }}}{d t} \text { и } \frac{d m 2_{\text {ИС }}}{d t} \\
& Q 1=\left(c 1_{\Gamma} \frac{d m 1_{\Gamma}}{d t}+c 1_{\text {Ж }} \frac{d m 1_{\text {Ж }}}{d t}\right) T 1_{K O H}, \\
& Q 1 "=\left(c 1_{\Gamma}\left(\frac{d m 1_{\Gamma}}{d t}-\frac{d m 1_{K O H}}{d t}\right)+\right. \\
& \left.+c 1_{Ж}\left(\frac{d m 1_{Ж}}{d t}+\frac{d m 1_{K O H}}{d t}\right)\right) T 1_{K O H}
\end{aligned}
$$




$$
\begin{aligned}
& \Delta Q 1=Q 1-Q 1^{\prime \prime}=\left(c 1_{\Gamma}-c 1_{Ж}\right) \frac{d m 1_{K O H}}{d t} T 1_{K O H}(8) \\
& Q 2=\left(c 2_{\Gamma} \frac{d m 2_{\Gamma}}{d t}+c 2_{Ж} \frac{d m 2_{Ж}}{d t}\right) T 2_{\text {исп }} \\
& Q 2 "=\left(c 2_{\Gamma}\left(\frac{d m 2_{\Gamma}}{d t}-\frac{d m 2_{\text {ИСП }}}{d t}\right)+\right.
\end{aligned}
$$

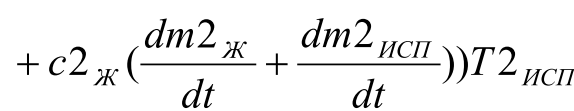

$$
\begin{aligned}
& \Delta Q 2=Q 2-Q 2^{\prime \prime}=\left(-c 2_{Г}+c 2_{\text {ж }}\right) \frac{d m 2_{\text {исп }}}{d t} T 2_{\text {исп }} \\
& \Delta Q_{T \Pi}=s k\left(T 1_{\text {КОН }}-T 2_{\text {ИСП }}\right)
\end{aligned}
$$

Из схемы тепловых потоков и соотношений (8) получим

$$
\begin{aligned}
& \Delta Q 1=-\Delta Q_{T \Pi}+\Delta Q 1_{K O H} \\
& \Delta Q 2=\Delta Q_{\text {тп }}-\Delta Q 2_{\text {исп }} \\
& \left(c 1_{\Gamma}-c 1_{Ж}\right) \frac{d m 1_{K O H}}{d t} T 1_{K O H}=
\end{aligned}
$$

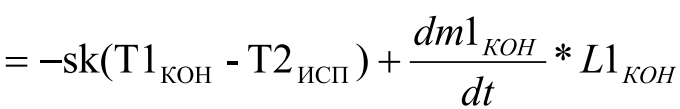

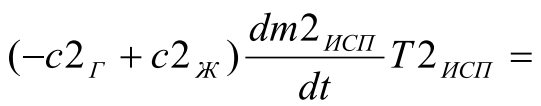

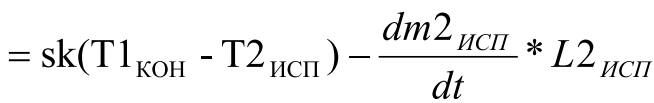

Из выражения (6) можно получить систему двух уравнений относительно неизвестных

$$
\begin{aligned}
& \frac{d m 1_{К О Н}}{d t} \text { и } \frac{d m 2_{\text {ИСП }}}{d t} . \\
& \left(\left(c 1_{\Gamma}-c 1_{Ж}\right) T 1_{K O H}-L 1_{K O H}\right) \frac{d m 1_{K O H}}{d t}= \\
& =-\operatorname{sk}\left(\mathrm{T}_{\text {КОН }}-\mathrm{T} 2_{\text {исп }}\right) \\
& \left(\left(-c 2_{\Gamma}+c 2_{\text {ж }}\right) T 2_{\text {иСп }}+L 2_{\text {исп }}\right) \frac{d m 2_{\text {иСП }}}{d t}= \\
& =\operatorname{sk}\left(\mathrm{T}_{\text {кон }}-\mathrm{T} 2_{\text {исп }}\right)
\end{aligned}
$$

Решая эту систем уравнений, получим

$$
\begin{aligned}
& \frac{d m 1_{\text {КО }}}{d t}=\frac{-\mathrm{sk}\left(\mathrm{T} 1_{\text {КОН }}-\mathrm{T} 2_{\text {ИСП }}\right)}{\left(c 1_{\Gamma}-c 1_{\text {Ж }}\right) T 1_{\text {КОН }}-L 1_{\text {КОН }}} \\
& \frac{d m 2_{\text {ИСП }}^{d t}}{d t}=\frac{\operatorname{sk}\left(\mathrm{T} 1_{\text {КОН }}-\mathrm{T} 2_{\text {ИСП }}\right)}{\left(-c 2_{\Gamma}+c 2_{\text {Ж }}\right) T 2_{\text {ИСП }}+L 2_{\text {ИСП }}}
\end{aligned}
$$

В данном случае в результате расчета мы получил приращения масс газа и жидкости в каналах теплообменника необходимые для перехода к расчету на новом участке.

\section{Зак^ючение}

В результате предложенных вычислений мы получили механизм определения параметров падения температуры и уменьшения или увеличения массы для всех возможных сочетаний фазовых переходов. Таким образом, можно построить диаграммы температуры и расхода жидкой и газообразной фаз на всём протяжении теплообменника при условии, что мы задали состояние (температура, расход и давление) на одном краю теплообменника. Так как в реальности для теплообменника с противотоком на одном краю находится вход одного контура и выход другого, то необходимо провести итеративную процедуру поиска решения аналогичную реализованной в алгоритме, предложенном для однофазного теплообменника [х, с. 35].

При построении модели многофазного теплообменника условиями выбора конкретной математической модели участка теплообменника служат достижения граничных значений фазовых переходов. Например, полному испарению соответствует состояние, когда расход жидкой фазы полностью достиг нуля, а расход газообразной фазы стал равен полному расходу через данный канал теплообменника. Это означает, что следующий элементарный участок будет соответствовать режиму нагрева газообразной фазы. Аналогично, если газообразная фаза остывает до температуры испарения - конденсации при заданном давлении в канале, то, соответственно, на соседнем элементарном участке должен начаться процесс конденсации в этом канале без изменения температуры, который будет продолжаться до полного перехода газообразной фазы в жидкую. После чего, на очередном элементарном участке начнётся процесс остывания уже жидкой фазы.

Итеративная процедура расчёта необходима только в случае моделирования теплообменника с противотоком. При параллельном потоке результат расчета получается за один цикл, т.к. оба исходных условия входные температуры нагревательного и нагреваемого каналов задаются на одном сечении теплообменника.

В результате сформирован метод расчёта процессов в теплообменных аппаратах, позволяющий учитывать фазовые переходы в процессе теплообмена. 


\section{ЛИТЕРАТУРА}

1. Алпаров А.У., Благов А. Е., Дегтярев Г. Л., Маханько А. А., Маханько А. В., Руденко С. А., Харитонов А. Ю. Микропроцессорная система управления самоходной моделью тримарана. Вестник Казанского государственного технического университета им. А. Н. Туполева. 2014. № 3. С. 197-200.

2. Ботов М.И., Давыдов Д. М., Давыдов А. М., Зиборов Д. М. Методика расчета теплообменников листоканального типа, применяемых в тепловых аппарата предприятий питания. Вестник Воронежского государственного университета инженерных технологий. 2019. № 1 (79). С. 53-58.

3. Голованчиков А.Б., Анцыперов С. А., Воротнева С. Б. Расчет теплообменников с учетом продольной теплопроводности по газовому теплоносителю. Повышение эффективности процессов и аппаратов в химической и смежных отраслях промышленности. Сборник научных трудов Международной научно-технической конференции, посвящённой 105-летию со дня рождения А. Н. Плановского. 2016. С. 207-210.

4. Голованчиков А.Б., Воротнева С. Б. Моделирование работы двухтрубного теплообменника с учетом теплодиффузии газового теплоносителя. «Химия, химическая технология», Т. 58, вып.9, 2015. С. 58-62.

5. Маханько А.В., Маханько А. А. (2017) Интерактивный метод расчета теплообменных аппаратов. Вопросы технических наук в свете современных исследований. Сборник статей по материалам IV международной научно-практической конференции. 2017. С. 34-42.

6. Соколова Г.П. (2017). Роль дисциплины гидравлика в фундаментальной подготовке инженерных специалистов. Казанский педагогический журнал. 2017. № 6 (125). C. 96-99.

7. Фарахов Т.М., Афанасьев Е. П., Лаптев А. Г. Расчет теплообменников с учетом структуры потоков. Вестник Ивановского государственного энергетического университета. 2019. С. 11-17.

8. Alekseev R.A., Kostukov A.V., Makarov A. R., Merzlikin V.G. (2016) Simulation of characteristics of thermo-hydraulic process in porous-net matrix of rotary heat exchanger. Global Journal of Pure and Applied Mathematics. Volume 12, Issue 4, 2016, Pages 2829-2838

9. Bokov A.V., Korytova M. A., Samarov A. B. (2019) Numerical modelling of convective heat and mass transfer in spherical coordinates. Bulletin of the South Ural State University, Series: Mathematical Modelling, Programming and Computer Software Volume 12, Issue 1, February 2019, Pages 96-109

10. Intensification of a laminar flow in a narrow microchannel with single-row inclined oval-trench dimples / S. A. Isaev, P. A. Baranov, A. I. Leontev, I. A. Popov // Technical Physics Letters. - 2018. - T. 44, № 5. - C. 398-400.

(c) Маханько Анатолий Васильевич, Маханько Андрей Анатольевич (andmahanko@mail.ru),

Соколова Галина Павловна ( glnsokolova@mail.ru ), Благов Алексей Евгеньевич ( bae311265@mail.ru ).

Журнал «Современная наука: актуальные проблемы теории и практики»

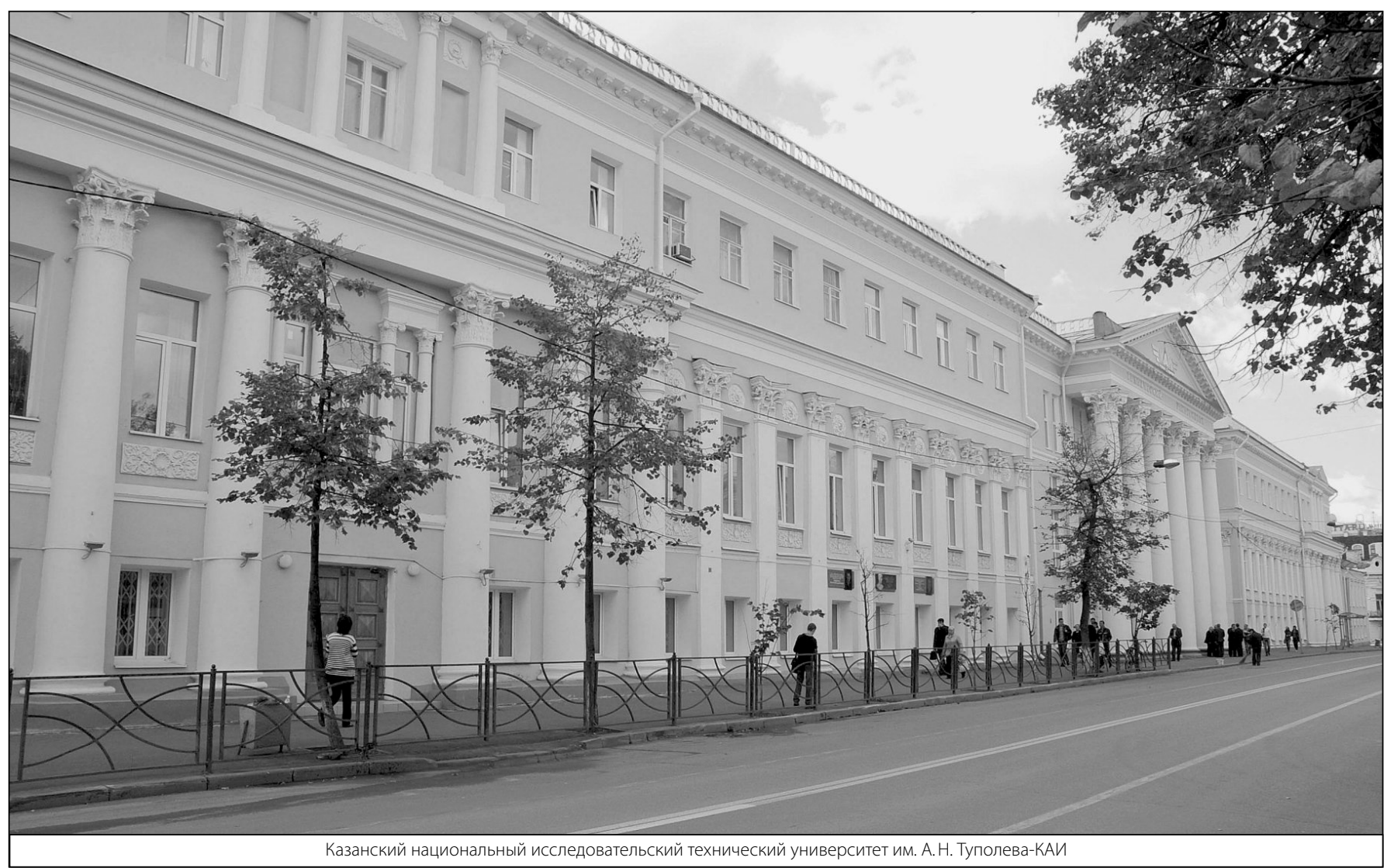

